\title{
O El "alma" de Métraux. La experiencia íntima de un etnógrafo poseído
}

\author{
The "soul" of Métraux. The intimate experience of a possessed \\ ethnographer \\ Jean-Pierre Goulard* \\ *CERMA/Mondes Américains, París
} RMA

\begin{abstract}
Resumen
El artículo reconstruye la trayectoria antropológica de Alfred Métraux (1902-1963) bajo la forma de un itinerario intelectual que se inicia con su crianza argentina y prosigue con su formación temprana en Francia y Suecia bajo la tutela de sus maestros Marcel Mauss y Erland Nordenskiöld, y luego con sus sucesivos desempeños institucionales en la Argentina, los Estados Unidos y Francia. A la luz de los estudios contemporáneos que revalorizan la figura disciplinar de Métraux, el texto recapitula aspectos específicos de su biografía, su personalidad y su obra, y analiza su prueba iniciática en el terreno, la fase posterior del análisis sintético y la etapa madura de compromiso en la ONU y la UNESCO. Finalmente, se presenta un breve manuscrito inédito de Métraux, realizado en 1928 para el diario argentino La Razón.
\end{abstract}

Palabras clave: Alfred Métraux; Antropología; Etnografía; Etnología; Historia del americanismo.

\begin{abstract}
The article portrays the anthropological trajectory of Alfred Métraux (1902-1963) as an intellectual itinerary that starts with his Argentinean upbringing and continues with his early anthropological training in France and Sweden under the tutelage of his teachers Marcel Mauss and Erland Nordenskiöld, and then with his successive institutional assignments in Argentina, the United States and France. Bearing in mind the contemporary studies on Métraux's biography, the paper analyzes his personality and his work, his initiatory ordeal in fieldwork, the later stage of analytical synthesis, and his mature stage of engagement at the UN and UNESCO. Finally, a short unpublished manuscript by Métraux, written in 1928 for the Argentine newspaper La Razón, is presented.
\end{abstract}

Keywords: Alfred Métraux; Anthropology; Ethnography; Ethnology; History of Americanism.

\section{Una "vida vagabunda" o "el viaje"}

En 1946, en sus intercambios epistolares con su "casi hermano" Pierre Verger, Alfred Métraux escribía que su amistad se debía a la "vida vagabunda" que ambos compartían (Verger, 1992, p. 177). En 1955 evocaba de la misma forma el "viaje" que cada uno de ellos había emprendido. De hecho, ¿qué mejor calificativo para su trayectoria que el de "errante", al que reivindica todavía ese año en otra carta a Michel Leiris? En una conferencia en Puerto Príncipe, este último evocaba en 1948 la noción de "encrucijada" o "cruce de caminos". Si, para Leiris, el "cruce" era "una conjunción de elementos distintos de la que nace la poesía" hecha de hibridación y sincretismo, ¿cómo no percibir también en él la coherencia de la trayectoria de Métraux? Leiris confirmaría más tarde la percepción que tenía de su amigo, evocando a "ese hombre [...] totalmente inadaptado a la vida actual y que vagó por todas partes sin jamás llegar a estar satisfecho: era realmente algo poético" (Leiris, cit. en Poitry 19951996, pp. 4, 8).
Si bien resulta fácil rastrear esos "cruces" en la trayectoria de Métraux, debe plantearse también la cuestión de los caminos recorridos desde cada uno de ellos al siguiente. En efecto, la vida de Alfred Métraux alternó períodos de intereses e investigaciones diferenciadas que, al mismo tiempo, estaban ligados entre sí. Aquí enfocaremos la atención en esos diversos espaciostiempos y examinaremos las temporalidades, postulando al hacerlo que los años que precedieron a su larga estadía en la Argentina, a partir de 1928, influenciaron de forma decisiva tanto en el plano intelectual como profesional a aquellos otros que siguieron hasta su propia desaparición, en 1963.

\section{El tiempo de la Argentina}

Puede proponerse el período de formación de Métraux como la primera temporalidad. Lo conocemos a grandes rasgos, pero debemos destacar algunos aspectos que servirán para entender cabalmente su trayectoria posterior. Se sabe que el joven Métraux y su familia 
acompañaron a su padre a la Argentina, donde se había instalado como médico en la ciudad de Mendoza'. Vivió su infancia en un entorno social del que habló muy poco después, contentándose con furtivos recuerdos. Se decía que montaba a caballo junto a niños indígenas, lo que bien puede ser cierto: tras la "Conquista del desierto" (18781885), las poblaciones nativas de la región habían sido diezmadas y, si bien la mayor parte de los sobrevivientes araucanos fueron desplazados y entregados a los nuevos terratenientes del espacio conquistado, o incluso sometidos a la esclavitud, otros lograron instalarse en la periferia de varias ciudades, entre ellas Mendoza. Escribe el propio Métraux: "Algunos grupos de estos indios fueron relocalizados en esta región tras la conquista del desierto llevada a cabo por el general Roca, cuyo resultado fue el brutal exterminio de los antiguos dueños del desierto. Aquellos que vivían en la provincia de Mendoza recibieron un trato particularmente cruel. Los sobrevivientes de estas horrendas y salvajes matanzas fueron dispersados en la provincia y entregados a diversas personas como sirvientes o, mejor dicho, como esclavos" (1929: 8).

Si bien no conocemos cuáles fueron las relaciones exactas entre padre e hijo, las jerarquías íntimas se construyen en la infancia. De tradición protestante, su padre no pudo dejar de compadecerse por la desesperanza y la dura situación de esos indígenas -algunos de los cuales tal vez hayan sido sus pacientes-, y muy probablemente comentó el tema en presencia de su hijo cuando todavía era un niño. Notemos, también, que años más tarde Alfred hijo (puesto que ambos llevaban el mismo nombre) dedicaría su tesis doctoral a su padre y que, al cursar la secundaria, cuando residía en Lausana con su madre, su padre los visitó en varias oportunidades.

Tras su formación escolar en Lausana, Métraux ingresó en la École des Chartes de París. Ahí estableció lazos duraderos con algunos colegas, entre ellos Georges Bataille. En la década de 1920, de regreso en la Argentina, participó en diversas excavaciones arqueológicas y realizó su primera investigación etnográfica entre los huarpes de la región de Mendoza; es decir, entre los descendientes de una población indígena desplazada a la fuerza y en buena parte diezmada. Métraux (1929) publicó los resultados de esta investigación en su primer aporte a la Revista del Instituto de Etnología de la Universidad de Tucumán, que acababa de fundar, comenzando con la siguiente dedicatoria: "A mi padre, en recuerdo del viaje de 1922". Podemos postular, entonces, que la elección de aquel primer trabajo etnográfico se debió a una empatía desarrollada durante su infancia en Mendoza. Él mismo recordaba en 1953: "Tuve una niñez argentina, y la cordillera y la pampa seca forman parte

1 Su padre fue jefe de sección y cirujano en el Hospital de Infecciosas de Mendoza. La calle "Alfredo Métraux" de esta ciudad es un reconocimiento a sus actividades. En 2015, asimismo, se inauguró un importante centro de salud en Maipú, cerca de Mendoza, que también lleva su nombre. de mis recuerdos. Creo que la afición que desde muy joven siento por el paisaje argentino indica el origen de mi carrera" (Métraux, 1953, p. 357). No obstante, para emplear la expresión de Olivier Sacks, lo que acaso siempre lo atormentó fue "un problema con... el apego, la pertenencia y la seguridad" (Sacks, 2006, p. 295). De todos modos, su primer período parisino fue una época de descubrimiento intelectual y relacional. Tras un año en la École des Chartes la abandonó y se inscribió en la $5^{\mathrm{a}}$ sección de la École Pratique des Hautes Études (EPHE) y luego en la École Nationale des Langues Orientales. De alguna manera, el cosmopolitismo que caracterizaría toda su carrera aparece como una suerte de continuación de esa formación atípica y múltiple que lo nutrió.

De la enseñanza de Marcel Mauss, Métraux recordaría sus lecciones sobre el enfoque etnográfico. Lo oyó preguntarse, el 28 de mayo de 1923: "¿En qué medida, cuando uno canta, el alma no se refleja en el estado del cuerpo?". Queda sintetizado, aquí, el hombre fisiológico, el hombre psicológico y el hombre social que son tan sólo abstracciones, y surge el "hombre concreto" que "prefigura lo que será para Mauss la búsqueda del 'hombre total'. Un hombre enteramente implicado en la vida real, comprometido sociológica, psicológica y biológicamente" (Bert, 2012). De esta manera, el método etnográfico elaborado por Mauss en sus seminarios de la EPHE pudo servir de marco a los trabajos de campo que Alfred Métraux iba a emprender posteriormente. En 1922, el mismo Mauss también había desarrollado una reflexión sobre la dispersión de los objetos materiales del rito a lo largo de Australia. Para resolverla, proponía tomar en cuenta datos lingüísticos, históricos y antropológicos con la finalidad de construir una historia comparada de las sociedades. Recordemos que Métraux dedicó su tesis doctoral complementaria a "don Marcel Mauss, como muestra de respeto y agradecimiento". La posterior relación de Métraux con Erland Nordenskiöld completaría esa pluriformación. Si bien su interés por la erudición y el trabajo archivístico sin duda debían mucho a su estadía en la École des Chartes, la importancia otorgada a la arqueología y a la museografía en los inicios de sus trabajos de campo reflejan asimismo las relaciones privilegiadas con Nordenskiöld, mientras que su estadía en la École des Langues Orientales le permitió percatarse de la importancia de la lingüística.

\section{El tiempo de la prueba o la experiencia del terreno}

Podemos suponer, entonces, que Métraux partió hacia América del Sur con el "hecho total" de Mauss por un lado y con el "hecho técnico total" de Nordenskiöld por otro. De hecho, llegó a la Argentina con este doble enfoque "teórico" propuesto por el primero y "práctico" planteado por el segundo. Al llegar a Buenos Aires dio una entrevista al periódico La Razón. Tras su publicación, por temor de no haber sido correctamente entendido, redactó la nota que reproducimos en anexo, en la cual explicitaba su 
enfoque. Defendía la idea de que "los objetos necesitan un alma: ésta se les otorga por la documentación que los rodea, y en eso consiste la obra científica". Debemos entender los resultados de sus investigaciones de entonces en la Argentina bajo esa perspectiva. Esa breve nota también nos informa algo sobre el pensamiento del autor, que llegaba con una visión que distinguía "lo antiguo" de "lo moderno". El primer término se refería al estado original de las sociedades, y el segundo a su situación contemporánea. Sus primeras publicaciones ciertamente parecen inscribirse en el marco de las de Nordenskiöld, pero lo animaba asimismo la voluntad de restituir "un alma" a los objetos recolectados, para darles vida de nuevo y, finalmente, volver a encontrar una o varias sociedades o culturas originales. Métraux siguió en eso a Nordenskiöld, que justificaba su propio trabajo por el conocimiento y el testimonio que dejaría a la humanidad acerca de la existencia de esas culturas llamadas a desaparecer. Anticipaba, de alguna manera, algo que podría llamarse "antropología de salvataje" o "de emergencia". Sus primeros escritos dan una fuerte sensación de acumulación en una perspectiva neodifusionista, pero su objetivo era distinto. De hecho, había emprendido un camino que lo llevaría a encontrar a una o varias "civilizaciones", siguiendo de nuevo una de las propuestas de Mauss (Durkheim y Mauss, 1971 [1913]). Era "nordenskioldiano", por cierto, pero con una perspectiva que iba más allá del enfoque de su maestro al darle una visión maussiana a sus resultados. Sin embargo, en algo coincidían las propuestas de Mauss y de Nordenskiöld: la primacía otorgada al trabajo etnográfico -una lección que Métraux aprendió con creces.

Escribir lo más rápido posible y restituir lo que observaba de manera explícita era entonces para Métraux la manera de evitar lo indecible y permitir una reconstrucción del material recolectado. Durante el tiempo de la escritura, permanecía en empatía con la vivencia inmediata que lo impregnaba y no podía despejar de otra manera. Se trataba de una representación activa y no distanciada. Sin embargo, las publicaciones resultantes contienen tal suma de datos etnográficos y de "propuestas" que sólo podemos remitir a los trabajos de investigadores argentinos (Bossert, Braunstein, Córdoba, Villar...) que se interesaron recientemente por su obra, la descifran y la analizan, mientras la contextualizan en su época y la someten a la prueba del tiempo, pues trabajan ellos mismos con algunas de las poblaciones estudiadas por Métraux.

Sus trabajos de campo en la Argentina, en los países vecinos y en la Isla de Pascua constituyeron a la vez una fuerte experiencia personal, así como una confrontación con una "realidad" que tuvo dificultades en aceptar. Abrevando en los relatos de las investigaciones llevadas a cabo por Nordenskiöld veinte años atrás, probablemente esperaba encontrar sociedades autónomas, en total posesión de sus culturas. En la transcripción de sus apuntes de campo trasluce su "desesperanza" ante la situación de las poblaciones que visitaba. Las percibía en tal estado de degradación que se propuso por tarea salvar de ellas aquello que todavía podía salvarse. Se impuso, así, una obligación de memoria realizando una colecta intensiva tanto de objetos como de minuciosas notas sobre las prácticas rituales y cotidianas que presenciaba. Recolectaba, por cierto, objetos para elaborar su clasificación geográfica, continuando así con su proyecto que debía abarcar a todas las culturas indígenas de la Argentina y más allá pero, en varias oportunidades, cuando su dueño se negaba a desprenderse de él, lamentaba no haber podido intercambiar o comprar algún artefacto que le parecía importante para completar una colección.

En el transcurso de sus investigaciones, Métraux prestaba mucha atención a la situación "moderna" de las sociedades que documentaba. Sin embargo, había llegado a la Argentina obsesionado por una visión idealizada del mundo indígena, que había cambiado mucho desde los viajes de Nordenskiöld. Ante los elementos contradictorios de la situación se lanzó desesperadamente a la restitución inmediata de los datos colectados. No se dedicó directamente a analizarlos, sino a darlos a conocer para (re)construir una o varias "civilizaciones" -en el sentido maussiano- como las del Chaco, y buscando ponerlas en relación con las "civilizaciones" vecinas, e incluso con las de los Andes.

No pudo concluir su proyecto de identificar los rasgos de una "civilización" en el continente sudamericano. Tras varios años "paralizado", nos dice, insatisfecho por los resultados conseguidos hasta entonces, aprovechó una propuesta de Mauss y de Rivet para alejarse del terreno, aunque sin pensar en una partida definitiva. De hecho, volvería a la Argentina en 1939 junto con su hermano Guy, por medio de una beca Guggenheim.

De alguna manera, esta búsqueda de una "civilización" se hizo finalmente posible en la Isla de Pascua, un campo bien delimitado esta vez. Tampoco debemos olvidar su estadía entre los chipayas, plasmada en larguísimos artículos. Su lectura muestra que había emprendido entonces una investigación multidireccional: tenía la conciencia de haber llevado a cabo entre ellos una investigación "total" y, hacia el final de su vida, expresó el deseo de retomar sus escritos al respecto y reunirlos en un libro ${ }^{2}$

\section{El tiempo del análisis y de la síntesis: Honolulu y los Estados Unidos}

Tras su estadía en la Isla de Pascua, Métraux aceptó una invitación del Bishop Museum de Honolulu. Encontró allí un ámbito donde aterrizar, por así decirlo. Siempre

2 Los escritos chipayas de Métraux están siendo reunidos y se publicarán próximamente en castellano. 
poseído por su necesidad de escribir, publicó una primera obra en ese ámbito en 1941, L'île de Pâques, resultado de su investigación anterior. Por primera vez, realizó entonces un verdadero trabajo de síntesis de los materiales recolectados, al que seguirían muchos más.

Cuando más tarde se instaló en los Estados Unidos, encontró rápidamente a los partidarios de la escuela norteamericana de antropología culturalista. ¿Fue para él un descubrimiento? Con un conocimiento profundo de las propuestas de Mauss, encontraba la culminación o la expresión de aquello que no se había atrevido a escribir, probablemente por exceso de modestia: la noción de "área cultural". Cuando salió de la Argentina, pensando en (re)construir una o varias "civilizaciones", ¿estaba acaso tan alejado del culturalismo? Su encuentro con la etnología norteamericana, a la que aceptó de entrada, lo alentó en su propósito y multiplicó su energía para escribir trabajos de síntesis. La intuición de Mauss se había abierto camino, y Métraux la concretó. Se zambulló en los escritos de los autores de esta escuela con tanta pasión como algunos años antes había buscado datos en el terreno. Este encuentro lo estimuló intelectualmente. Formaba parte de una escuela que tuvo gran impacto. En la misma época colaboró y discutió con colegas que ya estaban recorriendo ese mismo camino. Se dedicó entonces a trabajos que culminarían con su larga e intensa participación en la redacción del Handbook of South American Indians, para el cual escribió numerosas entradas -hasta llegar a afirmar, en su autobiografía, que había redactado una gran parte del mismo (Métraux, 1953, p. 359).

Estos encuentros le enseñaron algo más. Entendía ahora la decepción sentida ante la "desaparición" de las sociedades que había estudiado, tanto en la Argentina como en la Isla de Pascua, como un proceso de aculturación. La noción de áreas culturales le era familiar, pero no así la de aculturación: fue para él un verdadero descubrimiento, una apertura, y pudo por fin abandonar sus temores, lo que tal vez facilitó su trayectoria posterior. A partir de ese momento rompió con el cuestionamiento personal que había manifestado en muchas oportunidades acerca de la desaparición de aquellas sociedades. La noción de aculturación le permitió comprender que las sociedades se transformaban, pero no morían. Se adaptaban y entonces tenían recursos para integrar nuevas influencias (las del mundo de los blancos principalmente), que encontraban un lugar en su cultura mediante algunas modificaciones, pero les permitían no obstante conservar su identidad y sus peculiaridades lingüísticas, rituales, etc.

\section{El tiempo del compromiso: la ONU y la UNESCO}

Estos múltiples encuentros fueron para Métraux otras tantas "aperturas", a la par que una forma de tomar en consideración un cierto relativismo. Su dolorosa denuncia de la desaparición de las sociedades ya no era desesperada. Con toda evidencia, los compromisos que asumió posteriormente fueron consecuencias de ese caminar intelectual. Métraux participó, se comprometió y dirigió proyectos de gran alcance. Entre 1948 y 1950 dirigió para UNESCO el proyecto del Valle de Marbial, en Haití, un programa que apuntaba a reducir la pobreza en el medio rural. Mientras tanto, en paralelo, llevaba a cabo una investigación sobre el vudú (que no estaba contemplada en su proyecto original) y acompañó a una población desamparada en un proyecto que hoy calificaríamos "de desarrollo". Su participación fue no obstante distanciada, sobre todo si se la compara con su actuar en la Argentina.

Entre 1950 y 1960 integró la UNESCO como jefe del nuevo departamento de ciencias sociales aplicadas, encargado del programa de estudio sobre las razas. Empezó entonces numerosos proyectos de investigaciones sobre las relaciones raciales, con la finalidad de "contribuir positivamente a la solución del problema racial" (Métraux, 1955: 6). Métraux se involucró en algunos de estos proyectos, pero de manera diferente. En la Argentina se había comprometido con un enfoque de reconocimiento. Al ocupar su puesto en la UNESCO, en 1950, se colocó a mayor distancia. Su compromiso ya era otro: promover el reconocimiento del hecho indígena y de las poblaciones abandonadas o desamparadas. Participó de numerosos programas humanísticos sobre las relaciones raciales, propuso otros programas para los cuales solicitó la colaboración de muchos colegas, y ampliaba su campo de acción. Bajo ese impulso se publicaron varias series de escritos, entre las que destacan obras como Races et Classes dans le Brésil rural de Charles Wagley (1952), Race et Histoire de Claude Lévi-Strauss, Les élites de couleur dans une ville brésilienne de Thales de Azevedo (1953) y Contacts de civilisation en Martinique et en Guadeloupe de Michel Leiris (1955).

\section{Palabras finales}

La trayectoria de Alfred Métraux debe entenderse a partir del desarrollo de su propia vida intelectual, que se construyó por etapas. A partir de un cuestionamiento personal se involucró en un proyecto científico que se amplió progresivamente. Tras la Segunda Guerra Mundial asumió una posición que lo llevaría a desarrollar esas investigaciones en el marco de la UNESCO. Había entendido que sus cuestionamientos iban más allá del marco local y que debían considerarse por tanto en un plano más general e incluso global. Al solicitar el apoyo de investigadores de diversos horizontes, planteaba así la cuestión de los modos de relacionamiento en diferentes contextos sociopolíticos y varios continentes.

Jubilado de la UNESCO, fue elegido como profesor de la EPHE en 1959. Pensó entonces en volver a hacer trabajo de campo en Paraguay con un grupo tupí, lo que no era sorprendente. De hecho, escribía a Yvonne Odon, 
en 1947, que "la etnografía militante me dio grandes alegrías y me convenció que difícilmente podría vivir sin ser etnógrafo" (cit. en Laurière, 2005, p. 8). Se trataba de un compromiso que reivindicó durante toda su carrera y que hoy, demasiado a menudo, es el único que se le atribuye. Él mismo, por otra parte, se encargó de afirmarlo, porque mientras ocupaba diferentes funciones en organismos internacionales no cesó nunca de realizar breves investigaciones de campo en diversos continentes, que para él eran una necesidad. Y, en efecto, nunca se preocupó por adoptar una posición teórica aun cuando varios de sus textos ofrecen orientaciones para que otros las desarrollen.

Si tuviéramos que resumir la trayectoria de Alfred Métraux, podríamos decir entonces que pasó primero por un largo período de iniciación (que incluye su tiempo de formación y sus experiencias de campo en la Argentina y los países vecinos), el cual involucró una relación afectiva. Se dispuso luego a poner en práctica el bagaje universitario inicial adquirido en Francia. Ante la situación de los indígenas, para él dramática, se obligó luego a escribir para evitar la pérdida de los datos que, según él, todavía era posible recolectar durante la década de 1930. Siguió luego un segundo período que puede dividirse en une etapa intelectual, durante la cual completó y afirmó su formación teórica en los Estados Unidos dándose tiempo para los análisis antropológicos, y en una etapa de compromiso con sus funciones o puestos en la ONU y la UNESCO. En otros términos, tras la iniciación y el rite de passage de sus experiencias en el terreno asumió su nuevo estatus integrando una comunidad de investigadores que le ofreció un lugar antes de emprender y dirigir proyectos científicos de gran alcance, cuya pertinencia sigue siendo hoy válida.

En términos que hacemos nuestros, Leiris (1969) terminaba así su evocación de Métraux retratando a "un poeta... alguien que quisiera lograr una absoluta comprensión de aquello donde vive y romper su aislamiento comunicando esta comprensión". No queda nada que agregar.

\section{Bibliografía}

Azevedo, Th. de (1953), Les élites de couleur dans une ville brésilienne. París: UNESCO.

Bert, J. F. (2012), "Par quoi se définit une religion 'élémentaire'?". Archives de sciences sociales des religions, 159, 171-201.

Bing, F. (1964), "Entretiens avec Alfred Métraux". L'Homme, 4 (2), 20-31.

Durkheim, É. y M. Mauss (1971 [1913]), “Nota sobre la noción de civilización", M. Mauss, Obras II. Institución y culto (pp. 265-268). Barral: Barcelona.
Laurière, Ch. (2005), "D'une île à l'autre. Alfred Métraux en Haïti". Gradhiva, 1, 181-207.

Leiris, M. (1955), Contacts de civilisation en Martinique et en Guadeloupe. París: UNESCO.

Leiris, M. (1969), Cinq leçons d'ethnologie. Le Racisme et le Tiers-Monde. París: Denoël-Gonthier.

Lévi-Strauss, C. (1952), Race et Histoire. París: UNESCO.

Métraux, A. (1928), “Original del artículo de A. Métraux rectificando sus declaraciones publicadas en La Razón el 28 de noviembre de 1928", nota manuscrita, sd., 6 p.

Métraux, A. (1929), “Contribution à l'ethnographie et à I'archéologie de la Province de Mendoza (R.A.)", Revista del Instituto de Etnología de la Universidad de Tucumán, 1, Entrega 1, 5-73.

Métraux, A. (1953), "Autobiografía", Humanitas, 2 (4), 357-260.

Métraux, A. (1955), "Préface ", M. Leiris, Contacts de civilisation en Martinique et en Guadeloupe. París: Unesco-Gallimard.

Métraux, A., (2014 [1928]), La religion des Tupinamba, París: PUF.

Poitry, G. (1995-1996), "Carrefour des poètes : Michel Leiris et Alfred Métraux", Bulletin du Centre Genevois d'Anthropologie, 5, 3-9.

Sacks, O. (2016), En mouvement, Une vie, París: Seuil.

Verger, P. (1992), "Trente ans d'amitié avec Alfred Métraux, mon presque jumeau", Présence d'Alfred Métraux (pp. 173-191), París: UNESCO, París.

Wagley, Ch. (1952), Races et Classes dans le Brésil rural, París: UNESCO.

\section{ANEXO}

\section{Original del artículo de Alfred Métraux rectificando sus declaraciones publicadas en La Razón el $\mathbf{2 8}$ de noviembre de $1928^{3}$}

En la entrevista que tuve el honor de conceder a La Razón aparece una frase que, mal interpretada, podría dar una idea de mi pensamiento muy distinta de la que quise dar, más aún cuando se omitió el homenaje que, como extranjero, había hecho a las instituciones científicas del país.

3 Copia del manuscrito gentilmente cedida por Sophie Fischer. Se trata de la transcripción literal de aquello que parece ser el borrador de una respuesta a la publicación de la entrevista concedida por Métraux a La Razón, publicada poco tiempo después de llegar a Buenos Aires. 
La etnografía es una ciencia antigua en la Argentina. Se inició en el tiempo de la conquista con los relatos de viajes y, cuando se constituyó la República Argentina, revistió un carácter netamente científico. La primera muestra fue la publicación de colecciones completas como la colección De Angelis. Más tarde, bajo el impulso de quienes unían el amor por la ciencia al temple de los hombres de acción, se formaron grandes instituciones cuya máxima expresión fueron el Museo de Ciencias Naturales y el Museo de La Plata. La fama de estos institutos alcanzó al mundo entero por medio de espléndidas publicaciones como la Revista del Museo de La Plata y los Anales del Museo de La Plata. Pocos años después, se agregó el Museo de Etnología de la Facultad de Filosofía y Letras, ofreciendo recursos para las investigaciones de las antigüedades del país.

Estas pocas líneas son apenas un esbozo histórico, resumiendo casi cien años de esfuerzos y sobre todo de entusiasmo.

Veamos cómo están las cosas. Luego de su creación, el Museo ofrece en cuatro salas la sucesión de todas las antiguas culturas indígenas de la Argentina. Entre ellas están particularmente bien representadas las diversas industrias del Noroeste argentino: la famosa cultura calchaquí o diaguita, y con toda razón. Representa el máximo nivel de civilización logrado por el hombre en la Argentina antes de la llegada de los blancos, y debía ser homenajeada.

A su lado, vemos los restos materiales de lo que fueron las culturas vecinas influenciadas o no por ella. La influencia peruana fue poderosa en la Argentina, y debía estar presente. Hasta dónde sé, por ejemplo, ningún museo en Europa ofrece tanta riqueza de vasijas de la cultura de Nazca. ¿Se cansó la vista? Pasemos ahora al piso superior donde, en una sala de grandes dimensiones, se escalonan las diversas culturas de los indígenas modernos en sus supervivencias. Desde la Tierra de Fuego pasamos por el Chaco, y luego más allá, hasta los guerreros del Tapajós en el corazón de Brasil.

El indígena norteamericano también está presente y atestigua los lazos que existían probablemente antaño entre sur y norte. Los objetos necesitan un alma: ésta se les otorga por la documentación que los rodea, y en eso consiste la obra científica. Aquí la obra es perfecta. Todas las excavaciones se llevaron a cabo rigurosa y sistemáticamente, y amplias informaciones las acompañan, parte de las cuales ya fueron publicadas en una edición de lujo. Son los archivos humanos que permanecerán y permitirán fechar la historia humana en la Argentina mucho antes de la conquista.

El Museo de Etnología no persigue un único objetivo científico y no descuida sus deberes como órgano de la Facultad, es decir su valor educativo. Por esta razón, al lado de las colecciones argentinas se organizaron dos salas más donde están representados los demás pueblos del mundo. Aquellos que conocen la situación de las civilizaciones del Pacífico apreciarán el valor de esta obra.

Todos conocen el Museo de La Plata. Su objetivo, su proyecto, son evidentes para cualquiera que lo visite. Su historia es tan antigua como su fama. Demasiada gente lo demostró, y mi modesto homenaje no puede agregar nada más. Recordemos simplemente que está actualmente en plena remodelación arquitectural y que su valor lujoso no tendrá nada que envidiar a la riqueza de sus colecciones.

También está el Museo Nacional, con sus grandes colecciones arqueológicas y etnográficas que completan los archivos humanos del país. Debería hablar también de los institutos de investigaciones geográficas, arqueológicas o históricas que colaboran en la obra común: el conocimiento del pasado argentino.

El objetivo que persigo al llegar a la Argentina es aportar mi modesta contribución a este trabajo pasado y presente, dedicándome a investigar a las tribus que se están civilizando en el Norte. Ojalá las colecciones y las observaciones que se recolecten puedan agregarse a las anteriores e incrementar el material etnográfico que ya existe en Tucumán.

Dr. Alfred Métraux 